\title{
Preparation and characterization of
}

\section{poly(N-methylpyrrole)/MoO $\mathrm{M}_{3}$ hybrid}

\section{composites}

Shortened tittle: Poly(N-methylpyrrole)/ $\mathrm{MoO}_{3}$ hybrid composites

Margarita Sanchez-Jiménez, ${ }^{1}$ Francesc Estrany ${ }^{1,2, *}$ and Carlos Alemán, 1,2,*

${ }^{1}$ Departament d'Enginyeria Química, Escola d'Enginyeria de Barcelona Est (EEBE), Universitat Politècnica de Catalunya, Av. d'Eduard Maristany 10-14, 08019 Barcelona,

\section{Spain}

${ }^{2}$ Center for Research in Nano-Engineering, Universitat Politècnica de Catalunya, Campus Sud, Edifici C', C/Pasqual i Vila s/n, Barcelona E-08028, Spain 


\begin{abstract}
Poly(N-methylpyrrole) $/ \mathrm{MoO}_{3}$ composites have been prepared by in situ anodic polymerization using a 75:25 acetonitrile:water solution with monomer and previously exfoliated $\mathrm{MoO}_{3}$ particles as generation medium. The incorporated $\mathrm{MoO}_{3}$ improves the ability to exchange charge reversibly of poly(N-methylpyrrole) by $47 \%$ due to the induced structural changes. Thus, $\mathrm{MoO}_{3}$ reduces the cross-linking and transforms the uniform and compact surface morphology of poly(N-methylpyrrole) into a more open structure with higher roughness. These trends facilitate the access and escape of dopant ions during oxidation and reduction processes, respectively, in the composite with respect to the polymer alone PNMPy.
\end{abstract}

Keywords: Conducting polymer; Electrochemical activity; Molybdenum trioxide;

Polypyrrole 


\section{INTRODUCTION}

Although poly(N-methylpyrrole) (PNMPy) and polypyrrole (PPy) only differ in the $\mathrm{N}$-methylation, their properties are remarkably different. For example, the oxidation potential of the former is higher than that of the latter. ${ }^{[1]}$ Furthermore, the electrochemical and electrical properties of PNMPy are very poor with respect to those of PPy ${ }^{[1-3]}$ which has been attributed to the higher cross-linking of the former. ${ }^{[3]}$ Thus, although PPy displays some irregularities in the inter-ring linkages, PNMPy tends to stabilize branched molecules with abundant irregularities. In spite of these limitations, some potential application for PNMPy have been explored during the last years, results evidencing their excellent capacity to detect neurotransmitters and narcotic drugs. ${ }^{[4,5]}$ Regarding to the energy storage applications, the most successful results have been achieved when PNMPy is combined with more electroactive materials, as for example copper nanoparticles ${ }^{[6]}$ or layers of poly(3,4-ethylenedioxythiophene) (PEDOT) ${ }^{[7]}$ a conducting polymer (CP) with outstanding properties. ${ }^{[8-12]}$ Amazingly, the electrochemical and capacitive properties of electrodes made of alternated layers of PEDOT and PNMPy (PEDOT/PNMPy/PEDOT) were significantly better than those of PEDOT alone and PEDOT/PPy/PEDOT layers. ${ }^{[7,13]}$

Among inorganic electrode materials, molybdenum trioxide $\left(\mathrm{MoO}_{3}\right)$ particles have been subject of increasing interest due to their high electrochemical activity and ecofriendly nature. ${ }^{[14,15]}$ Apart from their potential use in technological applications such as optical devices, catalysis and sensors, ${ }^{[16,20]}$ nanostructured $\mathrm{MoO}_{3}$ has also been proved as electrode material for energy storage devices, such as batteries ${ }^{[21,22]}$ and electrochemical capacitors. ${ }^{[23]}$ In practice, the utilization of alone $\mathrm{MoO}_{3}$ particles is hindered by its poor electric conductivity, which limits the specific capacitance, cyclic

stability and faradaic redox reaction kinetics. Nanocomposites with graphene ${ }^{[24]}$ or 
carbon nanotubes ${ }^{[25]}$ have been found to overcome such shortcoming, enhancing the conductivity and improving the electrochemical performance of $\mathrm{MoO}_{3}$-based electrodes.

Another recently explored strategy is the hybridization of $\mathrm{MoO}_{3}$ with $\mathrm{CPs}$, which act as the conductive support. Within this context, $\mathrm{MoO}_{3} / \mathrm{PPy}$ hybrid nanocomposites have been prepared by wrapping homogeneous $\mathrm{CP}$ layers around $\mathrm{MoO}_{3}$ nanobelts to improve the capacitance of the inorganic material. ${ }^{[26,28]}$ Similarly, nanocomposites with PEDOT $^{[29-31]}$ and polyaniline ${ }^{[32,33]}$ (PAni) were prepared using different approaches. More specifically, $\mathrm{MoO}_{3} / \mathrm{PEDOT}^{[29-31]}$ nanocomposites were obtained by in situ intercalative polymerization methods, which interleaved PEDOT nanosheets between the inorganic layers, to enhance the capacitance of pristine $\mathrm{MoO}_{3},{ }^{[29-31]}$ while branched $\mathrm{MoO}_{3} / \mathrm{PAni}$ heteronanostructures were constructed by perpendicularly growing PAni nanorods from the surface of inorganic nanorods. ${ }^{[32,33]}$

The aim of this work is the opposite of the works previously reported for $\mathrm{MoO}_{3} / \mathrm{CP}$ nanocomposites. ${ }^{[26,33]}$ Thus, the main objective of this study is not to improve the properties of $\mathrm{MoO}_{3}$ but to promote the use of PNMPy as electrode material for energy storage devices by adding a small amount of $\mathrm{MoO}_{3}$ particles to improve the electrochemical properties of the CP. For this purpose, the electrical, electrochemical, and morphological properties of $\mathrm{PNMPy} / \mathrm{MoO}_{3}$ composites, which were prepared by in situ anodic polymerization, have been characterized. Results indicate that the incorporation of $10 \% \mathrm{w} / \mathrm{w} \mathrm{MoO}_{3}$, after exfoliation, improves significantly the electrochemical activity PNMPy. Inorganic particles, which are mainly located inside the polymeric matrix, alter the very uniform and compact structure of the latter $\mathrm{CP}$ opening and facilitate the exchange of ions with the environment during redox processes. 


\section{METHODS}

Materials. N-Methylpyrrole (NMPy) and acetonitrile were purchased from Aldrich and used as received. Anhydrous $\mathrm{LiClO}_{4}$, analytical reagent grade from Aldrich, was stored in an oven at $80{ }^{\circ} \mathrm{C}$ before use in the electrochemical trials. Powdered molybdenum (VI) oxide $\left(\mathrm{MoO}_{3}\right)$ was purchased from Aldrich and exfoliated as indicated below. Deionized water was used in all synthetic processes.

Preparation of PNMPy films. All systems studied in this work were prepared with a PGSTAT101 AUTOLAB potenciostat-galvanostat connected to a PC computer controlled through the NOVA 1.6 software, using a three-electrode one compartment cell under nitrogen atmosphere $\left(99.995 \%\right.$ in purity) at $25^{\circ} \mathrm{C}$.

PNMPy films were prepared by chronoamperometry (CA) under a constant potential of $1.40 \mathrm{~V}$. The compartment was filled with $50 \mathrm{~mL}$ of the generation medium, which consisted on a 75:25 acetonitrile:water solution with $10 \mathrm{mM}$ NMPy and $0.1 \mathrm{M} \mathrm{LiClO}_{4}$. Steel AISI 316 sheets of $4 \mathrm{~cm}^{2}$ area were employed as working and counter electrodes. The reference electrode was an $\mathrm{Ag} \mid \mathrm{AgCl}$ electrode containing a $\mathrm{KCl}$ saturated aqueous solution $\left(\mathrm{E}^{0}=0.222 \mathrm{~V}\right.$ vs. standard hydrogen electrode at $\left.25^{\circ} \mathrm{C}\right)$. The polymerization time, $\theta$, was kept fixed at $180 \mathrm{~s}$.

Preparation of PNMPy/MoO $\mathrm{M}_{3}$ films. The procedure and general conditions used to prepare the composite films were identical to that described for $\mathrm{CP}$ films with the obvious exception that $\mathrm{MoO}_{3}$ was added to the generation medium after exfoliation. For this purpose, a suspension of $10 \%$ w/w $\mathrm{MoO}_{3}$ (dry weight referred to the NMPy monomer concentration in the generation medium) in $25 \mathrm{~mL}$ of 75:25 acetonitrile:water was stirred during $1 \mathrm{~h}$ with a magnetic bar. After this, the exfoliated $\mathrm{MoO}_{3}$ suspension (25 mL) was added to $25 \mathrm{~mL}$ of the generation medium (i.e. $10 \mathrm{mM} \mathrm{NMPy}$ and $0.1 \mathrm{M}$ 
$\mathrm{LiClO}_{4}$ in 75:25 acetonitrile:water). The suspension and the solution were mixed in the cell and stirred 10 min before the start of electrochemical polymerization.

Electropolymerization kinetics. The kinetics of the anodic polymerization processes using static conditions was investigated considering different polymerization times. The weight of the insoluble polymer films $(m)$ for every polymerization time was determined as the difference between the masses of the coated and uncoated electrodes using a CPA26P Sartorius analytical microbalance with a precision of $2 \times 10^{-6} \mathrm{~g}$. Polymeric samples were obtained by scratching their deposits from the working steel electrode. The resulting powders were placed in a filter, rinsed with bi-distilled water and ethanol, and subsequently dried under vacuum conditions for $24 \mathrm{~h}$. The slope of linear plot representing the variation of $m$ against the charge consumed in the polymerization processes $\left(Q_{p o l}\right.$, in C) corresponds to the current productivity, which expressed as milligrams of electrogenerated polymer per coulomb of charge consumed during the anodic polymerization.

Characterization. The density of PNMPy and $\mathrm{PNMPy} / \mathrm{MoO}_{3}$ samples was determined by the flotation method using $\mathrm{CCl}_{4}: \mathrm{C}_{2} \mathrm{H}_{5} \mathrm{I}$ mixtures, in which they are insoluble.

The diameter of exfoliated $\mathrm{MoO}_{3}$ particles in 75:25 acetonitrile:water was determined at room temperature by dynamic light scattering (DLS). Measurements were performed with a NanoBrook Omni Zeta Potential Analyzer from Brookheaven Instruments Corporation.

Electrochemical properties were determined by cyclic voltammetry (CV) using an acetonitrile solution with $0.1 \mathrm{M} \mathrm{LiClO}_{4}$. The initial and final potentials were $-0.5 \mathrm{~V}$, the reversal potential was $1.6 \mathrm{~V}$, and the scan rate was $100 \mathrm{mV} / \mathrm{s}$. For all samples, the number of oxidation-reduction cycles applied was over 100. The ratio between the 
charge passed through the sample for every CV cycle and the mass the film obtained during the polymerization process was expressed as:

$$
\frac{\text { charge CV cycle }(\mathrm{C})}{\text { polymerization charge }(\mathrm{C})} \cdot \frac{\text { polymerization charge }(\mathrm{C})}{\text { Film mass }(\mathrm{mg})}=\frac{\mathrm{Q}_{\mathrm{i}}}{\mathrm{Q}_{\mathrm{pol}}} \cdot\left(\frac{\mathrm{Q}_{\mathrm{pol}}}{\mathrm{m}}\right)\left(\frac{\mathrm{C}}{\mathrm{mg}}\right)
$$

where $Q_{i}$ is the voltammetric charge corresponding to the cycle $i$ (in C), $Q_{p o l}$ is the voltammetric charge in the polymerization process (in C) and $\left(Q_{p o l} / m\right)$ is the inverse of current productivity in the polymerization process (in $\mathrm{C} / \mathrm{mg}$ ).

The thickness of the films was estimated from the mass of polymer deposited in the electrode using the procedure reported by Schirmeisen and Beck (i.e. considering the polymerization charge consumed in the generation of the films and the current productivity). ${ }^{[34]}$ The volume of polymer deposited in the electrode was obtained with the values of the mass and the density of the films. The electrical conductivity was obtained using the sheet resistance method.

Atomic force miscroscopy (AFM) images were obtained with a Molecular Imaging PicoSPM using a NanoScope IV controller under ambient conditions. The tapping mode AFM was operated at constant deflection. The row scanning frequency was set to $1 \mathrm{~Hz}$. AFM measurements were performed on various parts of the films, which provided reproducible images similar to those displayed in this work. The scan window sizes used in this work were $5 \times 5 \mu \mathrm{m}^{2}$. The statistical application of the NanoScope Analysis software was used to determine the root mean square roughness $\left(\mathrm{R}_{\mathrm{q}}\right)$, which is the average height deviation taken from the mean data plane; the average roughness $\left(R_{a}\right)$, which is the arithmetic average of the absolute values of the collected roughness data point, and the maximum roughness height $\left(\mathrm{R}_{\max }\right)$, which is the maximum peak to lowest valley vertical distance within a single sample length.

Scanning electron microscopy (SEM) studies were performed to examine the surface morphology of PNMPy and $\mathrm{PNMPy} / \mathrm{MoO}_{3}$ films. Dried samples were placed in a 
Focussed Ion Beam Zeis Neon 40 scanning electron microscope operating at $3 \mathrm{kV}$, equipped with an EDX spectroscopy system.

\section{RESULTS AND DISCUSSION}

After trials with different solvents and ratios, we found that $\mathrm{MoO}_{3}$ forms stable suspensions in 75:25 acetonitrile:water mixtures, avoiding fast or even moderately fast ( $\leq 15 \mathrm{~min})$ sedimentation of the inorganic particles. The average effective diameter $\left(D_{\text {eff }}\right)$ of exfoliated $\mathrm{MoO}_{3}$ particles, as determined by DLS, was around $385 \mathrm{~nm}$, even though $D_{\text {eff }}$ ranged from 290 to $525 \mathrm{~nm}$ (Figure 1).

The kinetics for the oxidation and subsequent polymerization of NMPy in absence and presence of exfoliated $\mathrm{MnO}_{3}$ particles was studied by generating films under a constant potential of $1.40 \mathrm{~V}$ onto steel AISI 316 electrodes. Reproducible film weights, $m$ (in $\mathrm{mg}$ ), were always obtained for polymerization times comprised between 60 and $1000 \mathrm{~s}$ for PNMPy and between 120 and $420 \mathrm{~s}$ for $\mathrm{PNMPy} / \mathrm{MoO}_{3}$. The polymerization charge consumed in the process, $Q_{p o l}$ (in C), was calculated on each chronoamperogram. Figure 2, which represents $m$ against $Q_{\text {pol }}$, provides a linear profile with a correlation coefficient higher than 0.95 for the two systems. This linearity reflects a Faradic behaviour of these anodic polymerizations. The current productivity, which corresponds to the slope of the $m$ - $Q_{p o l}$ plot, increases from $0.513 \mathrm{mg} / \mathrm{C}$ for PNMPy to $0.669 \mathrm{mg} / \mathrm{C}$ for $\mathrm{PNMPy} / \mathrm{MoO}_{3}$ (i.e. 30.4\%). These values reflect the influence of $\mathrm{MoO}_{3}$ on the polymerization process: the increment of the current productivity that for the same polymerization charge injected to the polymer, more mass is electrogenerated when exfoliated $\mathrm{MoO}_{3}$ particles are introduced in the generation medium. Thus, $\mathrm{MoO}_{3}$ particles reduce the formation of linkages between polymer chains (i.e. the degree cross- 
linking decreases), explaining the structural differences between PNMPy and $\mathrm{PNMPy} / \mathrm{MoO}_{3}$ (see below).

Table 1 displays the density, thickness and electrical conductivity of PNMPy and $\mathrm{PNMPy} / \mathrm{MoO}_{3}$ films. Density values, which were obtained by flotation using mixtures of tetrachloroethylene and carbon tetrachloride, reflect the incorporation of the inorganic particles in the $\mathrm{PNMPy} / \mathrm{MoO}_{3}$ films, which caused an increment of $5.4 \%$ in the density of PNMPy films. Considering the densities of the two components (1.595 and $4.69 \mathrm{~g} / \mathrm{cm}^{3}$ for PNMPy and $\mathrm{MoO}_{3}$, respectively), the weight composition of PNMPy/ $\mathrm{MoO}_{3}$ corresponds to 97.2\% PNMPy and 2.8\% $\mathrm{MoO}_{3}$. As the content of $\mathrm{MoO}_{3}$ in the composite is very low, the thickness of PNMPy and PNMPy/MoO $\mathrm{O}_{3}$ films are practically the same. In spite of this, important morphological and topographical differences have been identified between the two systems, as discussed below.

The incorporation of $\mathrm{MoO}_{3}$ particles does not provoke any significant change in the electrical conductivity, values being very similar for PNMPy and $\mathrm{PNMPy} / \mathrm{MoO}_{3}(\mathrm{Table}$ 1). Although the electric conductivity of pristine $\mathrm{MoO}_{3}, \sim 10^{-5} \mathrm{~S} / \mathrm{cm},{ }^{[29]}$ is two orders of magnitude higher than that of PNMPy, $\sim 10^{-7} \mathrm{~S} / \mathrm{cm}$, the low concentration of inorganic particles in the composite does not facilitate the formation of conductive pathways. Thus, $\mathrm{MoO}_{3}$ particles aggregate forming clusters (see SEM discussion below), which are unconnected among them, rather than distribute homogeneously forming conduction paths. Consequently, the electrical conductivity measured for $\mathrm{PNMPy} / \mathrm{MoO}_{3}$ must be entirely attributed to the polymeric matrix.

In spite of $\mathrm{MoO}_{3}$ particles do not provide any advantage in terms of electrical conductivity, their incorporation improve considerably the electrochemical activity of the PNMPy. Control voltammograms of $\mathrm{PNMPy} / \mathrm{MoO}_{3}$ and $\mathrm{PNMPy}$ registered in acetonitrile with $0.1 \mathrm{M} \mathrm{LiClO}_{4}$ are compared in Figure 3a. The electroactivity, which 
corresponds to the similarity between the cathodic and anodic areas (i.e. the ratio between the reduction and oxidation charges) is $47 \%$ higher for the former than for the latter. Although this difference decreases to $31 \%$ when charges normalized per unit of produced film mass are considered (i.e. 44.14 and $33.81 \mathrm{C} / \mathrm{g}$, respectively), results indicate that the ability of PNMPy to exchange charge reversibly improves significantly upon the incorporation of a small amount of $\mathrm{MoO}_{3}$. This observation is fully consistent with the fact that the amount of cross-links is lower in PNMPy/MoO $\mathrm{O}_{3}$ and $\mathrm{PNMPy}$. The variation of the ratio between the charge passed through the film and its mass $(Q / m$, Eqn 1) against the number of oxidation-reduction cycles is displayed in Figure 3b. As it can be seen, initially the capacitive behaviour is significantly higher for $\mathrm{PNMPy} / \mathrm{MoO}_{3}$ than for PNMPy, which is consistent with the increment of electroactivity induced by the inorganic particles (Figure 3a). However, this synergistic effect becomes less pronounced with increasing number of cycles, the $Q / m$ ratio for the two systems being similar after 60 cycles. Thus, although for both systems the $Q / m$ ratio decreases with increasing number of cycles, such reduction is more marked for $\mathrm{PNMPy} / \mathrm{MoO}_{3}(43 \%)$ than for PNMPy (28\%). This feature suggests that $\mathrm{MoO}_{3}$ particles affect the structure PNMPy, facilitating the degradative effects typically produced by the application of consecutive oxidation-reduction cycles.

Figure 4 displays representative 2D height and 3D AFM images and SEM micrographs for PNMPy and PNMPy/MoO . As it can be seen, PNMPy films exhibit a uniform, globular and very compact surface morphology. This closed structure is consistent with a homogeneous distribution of the polymer chains. The incorporation of $\mathrm{MoO}_{3}$ provokes drastic changes in the topography and surface morphology. Thus, the corpuscles that define the globular morphology of PNMPy are less pronounced at the individual level and tend to organize in groups forming relatively large clusters. 
Topographic AFM images and SEM micrographs show that such clusters, which tend to present a rounded profile, are bounded by channels of micrometric length that appear to have a preferential orientation (Figure $4 b$ ). It is worth noting that the roughness (Table 1) of PNMPy films should be attributed to the marked individual corpuscles associated to the globular morphology, while the roughness of $\mathrm{PNMPy} / \mathrm{MoO}_{3}$, which is $\sim 45 \%$ higher, is mainly due by the apparition of channels separating clusters (i.e. different levelling of two distinctive topographic features). Both the increment in the roughness induced by $\mathrm{MoO}_{3}$ and the apparition of microchannels in the composite with respect to PNMPy are consistent with the higher electroactivity of the former. Accordingly, the distinctive surface features PNMPy/MoO 3 reflect a structure less closed than the one of PNMPy, which makes easier the access and escape of the ions into the polymeric matrix during the Faradaic processes. The morphologies displayed in Figure 4 are fully consistent with the reduction of cross-linking between polymer chains found in the composite.

Inspection of representative AFM phase images for PNMPy and $\mathrm{PNMPy} / \mathrm{MoO}_{3}$ (Figure 5a) indicates that, in general, the inorganic particles remain below the surface and are coating by the $\mathrm{CP}$. Although $\mathrm{MoO}_{3}$ particles are not observed at the surface of the composite phase images, the response of the $\mathrm{PNMPy} / \mathrm{MoO}_{3}$ is clearly different from that of PNMPy in terms of stiffness/softness and adhesion between the tip and the surface. Thus, the phase shifts to higher values in the composite reflecting that the amount of inelastic energy transferred from the tip to the sample surface increases when PNMPy coats $\mathrm{MoO}_{3}$ aggregates. The internal distribution of inorganic particles is consistent with representative SEM micrographs of $\mathrm{PNMPy} / \mathrm{MoO}_{3}$ films (Figure 4), while the tendency of such particles to aggregate in clusters is supported by a few SEM micrographs that display groups of polyhedral particles at the surface of the composite 
films (Figure 5b). It should be remarked that the location of particles onto the surface of the films was relatively infrequent and cannot be considered as a representative structural characteristic of the composite. Semi quantitative EDX analyses were carried out to corroborate that, when detected, such groups of particles correspond to $\mathrm{MoO}_{3}$, whereas the surface is exclusively formed by PNMPy (i.e. the small amount of Mo should be attributed to internal inorganic particles not observed in the micrograph).

SEM micrographs of PNMPy and PNMPy/MoO 3 after 100 consecutive oxidationreduction cycles are provided in Figure 6. Comparison of PNMPy micrographs displayed in Figures 4a and 6a evidences a change in the morphology of the $\mathrm{CP}$. Thus, the globular and compact morphology of pristine PNMPy transforms into a clustered morphology after apply 100 redox cycles. More specifically, the uniformly distributed globules of PNMPy, which are small (around 100-300 nm) and responsible of the locally homogeneous morphology characteristic of PNMPy, group forming relatively large clusters (around 2-4 $\mu \mathrm{m}$ ) and giving place to locally heterogeneous surfaces. This is reflected not only by the agglomeration of grouped globules but also by the apparition of deep trenches separating the agglomerates of clusters. In spite of this, the surface of PNMPy remains apparently compact, which explains that the access and escape of the dopant anion, which is difficult since the first Faradaic process (Figure 3b), only reduces moderately when the number of redox cycles increases (i.e. $Q / m$ decreases from $34 \mathrm{C} / \mathrm{g}$ to 29 and $24 \mathrm{C} / \mathrm{g}$ after 1 and 100 redox cycles, respectively; Figure 3b).

In addition of a clustering process similar to that described above for PNMPy, another two important structural changes are identified in $\mathrm{PNMPy} / \mathrm{MoO}_{3}$ films after the application of consecutive redox cycles. Firstly, local micrometric cracks appear at the composite surface (white circle and magnified inset in Figure 6b), this feature occurring in around $70 \%$ of the assayed films. In addition, the surface of the film is abundantly 
covered of nanoparticles, which are randomly and uniformly distributed onto the surface. The $D_{\text {eff }}$ of such nanoparticles is around $20 \mathrm{~nm}$, this value being one order of magnitude smaller than that of the exfoliated $\mathrm{MoO}_{3}$ particles used in the in situ polymerization $\left(D_{\text {eff }}=385 \mathrm{~nm}\right)$. Overall, these observations suggest that $\mathrm{MoO}_{3}$ agglomerates formed during the polymerization, which were confined inside the polymeric matrix, break into very small nanoparticles because of the charge transported during voltammetric scans. This process produces cracks at the polymeric films facilitating the segregation of such small nanoparticles towards the surface.

Figure 6c displays a high magnification backscattered SEM micrograph of PNMPy/ $\mathrm{MoO}_{3}$ after 100 redox cycles. In this micrograph the presence of different elements is evidenced by the contrast, which increases with the difference between the atomic weights of the elements contained in the material. The contrast of the nanoparticles with respect to the rest of the film confirms that they are constituted of molybdenum, which is the heaviest element of PNMPy/MoO3, whereas the matrix is made of elements with similar atomic weights (hydrogen, carbon and nitrogen).

\section{CONCLUSIONS}

In summary, we present an in situ polymerization approach to improve the electrochemical properties of PNMPy by preparing a composite with electroactive and eco-friendly $\mathrm{MoO}_{3}$ particles with $D_{\text {eff }}=385 \mathrm{~nm}$. The main characteristic of this composite is that $\mathrm{MoO}_{3}$ particles tend to aggregate inside the CP matrix and reduce the cross-linking between polymer chains. Although the concentration of $\mathrm{MoO}_{3}$ is too low $(2.8 \% \mathrm{w} / \mathrm{w})$ for the formation of conduction paths, the electrochemical activity of $\mathrm{PNMPy} / \mathrm{MoO}_{3}$ is significantly higher than that of pristine PNMPy due to the favourable synergy between the inorganic particles and the CP matrix. However, this improvement 
is retained by only $\sim 60$ oxidation-reduction cycles, the behaviour of two materials becoming very similar after such amount of redox processes. This limitation is due to the reduced structural stability of $\mathrm{MoO}_{3}$ particles, which segregate into very small nanoparticles $\left(D_{e f f} \approx 20 \mathrm{~nm}\right)$ upon the application of voltammetric scans. In spite of such segregation causes the formation of cracks at the surface of the CP film, after 60 cycles the electrochemical stability of $\mathrm{PNMPy} / \mathrm{MoO}_{3}$ is still very similar to that of PNMPy. This feature suggests that the dispersion of such small $\mathrm{MoO}_{3}$ particles onto the PNMPy surface still exerts the favourable synergy detected for the larger particles embedded into the matrix.

Overall, results indicate that $\mathrm{PNMPy} / \mathrm{MoO}_{3}$ is a promising alternative to pristine PNMPy. This is particularly relevant for applications associated to a relatively short cycle life and dominated by the intrinsic electrochemical behaviour of PNMPy, as for example selective biosensing. However, $\mathrm{PNMPy} / \mathrm{MoO}_{3}$ is not appropriated for applications requiring long term cyclability, as for example the fabrication of energy storage devices.

\section{ACKNOWLEDGEMENTS}

This work was supported by MINECO-FEDER (MAT2015-69367-R). Support for the research of C.A. was received through the prize "ICREA Academia" for excellence in research funded by the Generalitat de Catalunya.

\section{REFERENCES}

1. Oliver, R.; Muñoz, A.; Ocampo, C.; Alemán, C.; Estrany, F. Electrochemical characteristics of copolymers electrochemically synthesized from $\mathrm{N}$ - 
methylpyrrole and 3,4-ethylenedioxythiophene on steel electrodes: Comparison with homopolymers. Chem. Phys. 2006, 24, 299-306.

2. Aradilla,D.; Estrany, F.; Casellas, F.; Iribarren, J. I.; Alemán, C. Allpolythiophene rechargeable batteries. Org. Electr. 2014, 15, 40-46.

3. Alemán, C.; Casanovas, J.; Torras, J.; Bertran, O.; Armelin, E.; Oliver, R.; Estrany, F. Cross-linking in polypyrrole and poly(N-methylpyrrole): Comparative experimental and theoretical studies. Polymer 2008, 49, 10661075.

4. Marti, M.; Fabregat, G.; Estrany, F.; Alemán, C.; Armelin, E. Nanostructured conducting polymer for dopamine detection. J. Mater. Chem. 2010, 20, 1065210660.

5. Teixeira-Dias, B.; Alemán, C.; Estrany, F.; Azambuja, D. S.; Armelin, E. Microstructures of poly(N-methylpyrrole) and their interaction with morphine. Electrochim. Acta 2011, 56, 5836-5843.

6. Sánchez-Jiménez, M.; Estrany, F.; Alemán, C. Properties of Cu nanoparticlespoly(N-methylpyrrole) composites. Polym. Compos. 2016, 37, 594-601.

7. Aradilla, D.; Estrany, F.; Alemán, C. Symmetric supercapacitors based on multilayers of conducting polymers. J. Phys. Chem. C 2011, 115, 8430-8438.

8. Okuzaki, H.; Suzuki, H.; Ito T. Electromechanical properties of poly(3,4ethylenedioxythiophene)/poly(4-styrene sulfonate) films. J. Phys. Chem. B 2009, 113, 11378-11383.

9. Karlsson, R.H.; Herland, A.; Hamedi, M.; Wigenius, J. A.; Åslund, A.; Liu, X.; Fahlman, M.; Inganäs, O.; Konradsson, P. Iron-catalyzed polymerization of alkoxysulfonate-functionalized 3,4-ethylenedioxythiophene gives water-soluble 
poly(3,4-ethylenedioxythiophene) of high conductivity. Chem. Mater. 2009, 21, $1815-1821$.

10. Fabretto, M.V.; Evans, D.R.; Mueller, M. Zuber, K.; Hojati-Talemi, P.; Short, R.D.; Wallace, G.G.; Murphy, P.J. Polymeric material with metal-like conductivity for next generation organic electronic devices. Chem. Mater. 2012, 24, 3998-4003.

11. Mueller, M.; Fabretto, M.; Evans, D.; Hojati-Talemi, P.; Gruber, C.; Murphy, P. Vacuum vapour phase polymerization of high conductivity PEDOT: Role of PEG-PPG-PEG, the origin of water, and choice of oxidant. Polymer 2012, 53, 2146-2151.

12. Groenendaal, B.L.; Zotti, G.; Aubert, P.H.; Waybright, S.M.; Reynolds, J.R. Electrochemistry of poly(3,4-alkylenedioxythiophene) derivatives. Adv. Mater. 2003, $15,855-879$.

13. Aradilla, D.; Estrany, F.; Oliver, R.; Alemán, C. Properties of nanometric and micrometric multilayered films made of three conducting polymers. Eur. Polym. J. 2010, 46, 2222-2228.

14. Lou, X.W.; Zeng, H.C. Hydrothermal synthesis of $\alpha-\mathrm{MoO}_{3}$ nanorods via acidification of ammonium heptamolybdate tetrahydrate. Chem. Mater. 2002, $14,4781-4789$.

15. Yao, D.D.; Ou, J.Z.; Latham, K.; Zhuiykov, S.; O’Mullane, A.P.; Kalantarzadeh, K. Electrodeposited $\alpha$ - and $\beta$-phase $\mathrm{MoO}_{3}$ films and investigation of yheir gasochromic properties. Cryst. Growth Des. 2012, 12, 1865-.

16. Zhou, J.; Xu, N.S.; Deng, S.Z.; Chen, J.; She, J.-C.; Wang, Z.-L. Large-area nanowire arrays of molybdenum and molybdenum oxides: Synthesis and field emission properties. Adv. Mater. 2003, 15, 1835-1840. 
17. Li, X.L.; Liu, J.F.; Li, Y.D. Low-temperature synthesis of large-scale singlecrystal molybdenum trioxide $\left(\mathrm{MoO}_{3}\right)$ nanobelts. Appl. Phys. Lett. 2002, 81, $4832-4834$.

18. Alsaif, M.M.Y.A.; Latham, K.; Field, M.R.; Yao, D.D.; Medhekar, N.V.; Beane, G.A.; Kaner, R.B.; Russo, S.P.; Ou, J.Z.; Kalantar-zadeh, K. Tunable plasmon resonances in two-dimensional molybdenum oxide nanoflakes. Adv. Mater. 2014, 26, 3931-3937.

19. Chen, D.; Liu, M.; Yin, L.; Li, T.; Yang, Z.; Li, X.J.; Fan, B.B.; Wang, H.L.; Zhang, R.; Li, Z.X.; Xu, H.L.; Lu, H.X.; Yang, D.Y.; Sun, J.; Gao, L. Singlecrystalline MoO3 nanoplates: topochemical synthesis and enhanced ethanolsensing performance. J. Mater. Chem. 2011, 21, 9332-9342.

20. Balendhran, S.; Walia, S.; Alsaif, M.; Nguyen, E.P.; Zhen, J.; Zhuiykov, S.; Sriram, S.; Bhaskaran, M.; Kalantar-zadeh, K. Field effect biosensing platform based on 2D $\alpha-\mathrm{MoO}_{3}$. ACS Nano 2013, 11, 9753-9760.

21. Tao, T.; Glushenkov, A.M.; Zhang, C.; Zhang, H.; Zhou, D.; Guo, Z.; Liu, H.K.; Chen, Q.; Hu, H.; Chen, Y. $\mathrm{MoO}_{3}$ nanoparticles dispersed uniformly in carbon matrix: a high capacity composite anode for Li-ion batteries. J. Mater. Chem. 2011, 21, 9350-9355.

22. Chernova, N.A.; Roppolo, M.; Dillon, A.C.; Whittingham, M.S. Layered vanadium and molybdenum oxides: batteries and electrochromics. J. Mater. Chem. 2009, 19, 2526-2552.

23. Brezesinski, T.; Wang, J.; Tolbert, S.H.; Dunn, B. Ordered mesoporous alpha$\mathrm{MoO}_{3}$ with iso-oriented nanocrystalline walls for thin-film pseudocapacitors. Nat. Mater. 2010, 9, 146-151. 
24. Tang, Q.; Wang, L.; Zhu, K.; Shan, Z.; Qin, X. Synthesis and electrochemical properties of H-MoO3/graphene composite. Mater. Lett. 2013, 100, 127-129.

25. Mahmood, Q.; Yun, H.J.; Kim, W.S.; Park, H.S. Highly uniform deposition of MoO3 nanodots on multiwalled carbon nanotubes for improved performance of supercapacitors. J. Power Sources 2013, 235, 187-192.

26. Liu, Y.; Zhang, B.; Yang, Y.; Chang, Z.; Wen, Z.; Wu, Y. Polypyrrole-coated $\alpha$ $\mathrm{MoO}_{3}$ nanobelts with good electrochemical performance as anode materials for aqueous supercapacitors. J. Mater. Chem. A 2013, 1, 13582-13587.

27. Zhang, X.; Zeng, X.; Yang, M.; Qi, Y. Investigation of a branchlike $\mathrm{MoO}_{3} /$ polypyrrole hybrid with enhanced electrochemical performance used as an electrode in supercapacitors. ACS Appl. Mater. Interfaces 2014, 6, 11251130.

28. Liu, Y.; Zhang, B.H.; Xiao, S.Y.; Liu, L.L.; Wen, Z.B.; Wu, Y.P. A nanocomposite of $\mathrm{MoO}_{3}$ coated with PPy as an anode material for aqueous sodium rechargeable batteries with excellent electrochemical performance. Electrochim. Acta 2014, 116, 512-517.

29. Murugan, A.V.; Viswanath, A.K.; Gopinath, C.S.; Vijayamohanan, K. Enhancement of double-layer capacitance behavior and its electrical conductivity in layered poly (3, 4-ethylenedioxythiophene)-based nanocomposites. Appl. Phys. Lett. 2005, 87, 243511.

30. Murugan, A.V.; Viswanath, A.K.; Gopinath, C.S.; Vijayamohanan, K. Highly efficient organic-inorganic poly(3,4-ethylenedioxythiophene)-molybdenum trioxide nanocomposite electrodes for electrochemical supercapacitor. J. Appl. Phys. 2006, 100, 074319. 
31. Murugan, A.V. Novel organic-inorganic poly (3,4-ethylenedioxythiophene) based nanohybrid materials for rechargeable lithium batteries and supercapacitors. J. Power Sources 2006, 159, 312-318.

32. Kumar, V.; Lee, P.S. Redox active polyaniline-h-MoO3 hollow nanorods for improved pseudocapacitive performance. J. Phys. Chem. C 2015, 19, 90419049.

33. Wang, Q.; Lei, Z.; Chen, Y.; Ouyang, Q.; Gao, P.; Qi, L.; Zhu, C.; Zhang, J. Branched polyaniline/molybdenum oxide organic/inorganic heteronanostructures: synthesis and electromagnetic absorption properties. J. Mater. Chem. A 2013, 1, 11795-11801.

34. Schirmeisen, M.; Beck, F. J. Electrocoating of iron and other metals with polypyrrole. J. Appl. Electrochem. 1989, 19, 401-409. 
Table 1. Density $\left(\rho\right.$, in $\left.\mathrm{g} / \mathrm{cm}^{3}\right)$, thickness $(L$, in $\mu \mathrm{m})$, root mean square roughness $\left(\mathrm{R}_{\mathrm{q}}\right.$, in $\mathrm{nm})$, average roughness $\left(\mathrm{R}_{\mathrm{a}}\right.$, in $\left.\mathrm{nm}\right)$, maximum roughness $\left(\mathrm{R}_{\max }\right.$, in $\left.\mathrm{nm}\right)$ and electrical conductivity (S/cm) of PNMPy and PNMPy/MoO 3 films.

\begin{tabular}{ccc}
\hline & PNMPy & PNMPy/MoO \\
\hline$\rho\left(\mathrm{g} / \mathrm{cm}^{3}\right)$ & 1.595 & 1.682 \\
$L(\mu \mathrm{m})$ & $0.42 \pm 0.02$ & $0.44 \pm 0.02$ \\
$\sigma \cdot 10^{7}(\mathrm{~S} / \mathrm{cm})$ & $1.8 \pm 0.2$ & $1.2 \pm 0.2$ \\
$\mathrm{R}_{\mathrm{q}}(\mathrm{nm})$ & $103 \pm 14$ & $148 \pm 11$ \\
$\mathrm{R}_{\mathrm{a}}(\mathrm{nm})$ & $80 \pm 12$ & $117 \pm 11$ \\
$\mathrm{R}_{\max }(\mathrm{nm})$ & $659 \pm 96$ & $773 \pm 68$ \\
\hline
\end{tabular}




\section{CAPTIONS TO FIGURES}

Figure 1. Effective diameter $\left(D_{\text {eff }}\right.$, in $\left.\mathrm{nm}\right)$ as measured by DLS of exfoliated $\mathrm{MoO}_{3}$ particles in 75:25 acetonitrile:water.

Figure 2. Variation of weight $(m)$ of the $\mathrm{PNMPy} / \mathrm{MoO}_{3}$ or PNMPy films deposited on $4 \mathrm{~cm}^{2}$ steel electrodes from $10 \mathrm{mM}$ monomer in 75:25 acetonitrile:water solutions with $0.1 \mathrm{M} \mathrm{LiClO} 4$ at constant potential of $1.40 \mathrm{~V}$ against the consumed polymerization charge $\left(Q_{p o l}\right)$. The current productivity corresponds to the slope of the fitted straight line.

Figure 3. For PNMPy and $\mathrm{PNMPy} / \mathrm{MoO}_{3}$ films: (a) Control voltammograms registered at $25{ }^{\circ} \mathrm{C}$ in a $0.1 \mathrm{M} \mathrm{LiClO}_{4}$ acetonitrile solution; and (b) Variation of the ratio between the charge passed through the film and its mass ( $Q / m$ Eqn 1) against the number of consecutive oxidation-reduction cycles in a $0.1 \mathrm{M} \mathrm{LiClO}_{4}$ acetonitrile. Voltammograms were conducted using the following parameters: scan rate of 100 $\mathrm{mV} / \mathrm{s}$; initial and final potentials of $-0.50 \mathrm{~V}$; and reversal potential of $1.60 \mathrm{~V}$.

Figure 4. Representative 2D height and 3D topographic AFM images (top) and SEM micrographs (bottom) of (a) PNMPy and (b) PNMPy/MoO $\mathrm{M}_{3}$ films. The AFM image windows are $5 \times 5 \mu \mathrm{m}^{2}$ in all cases.

Figure 5. (a) 2D phase AFM images of PNMPy and $\mathrm{PNMPy} / \mathrm{MoO}_{3}$ films. Phase images correspond to the height images displayed in Figure 4. The AFM image windows are $5 \times 5 \mu^{2}$. (b) $\mathrm{SEM}$ micrograph displaying a group of $\mathrm{MoO}_{3}$ particles, which are rarely detected at the surface, and EDX elemental analysis of the sample at the indicated positions: PNMPy film surface (light gray) and inorganic particles (line).

Figure 6. SEM micrographs of (a) PNMPy and (b) $\mathrm{PNMPy}_{\mathrm{MoO}}$ after 100 consecutive oxidation-reduction cycles. (c) Backscattered micrograph of PNMPy/MoO 3 after 100 consecutive oxidation-reduction cycles. 


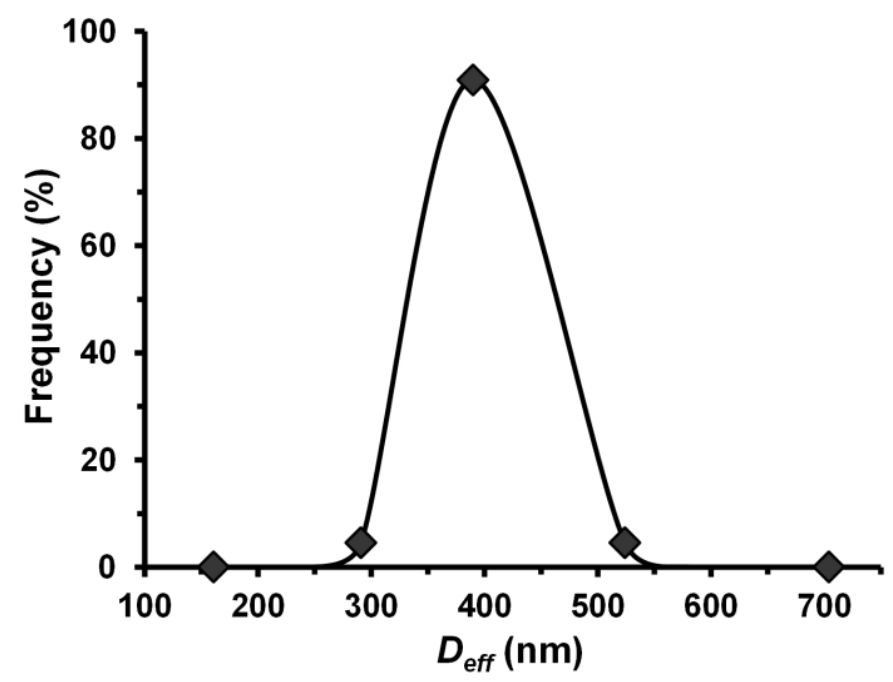

Figure 1 


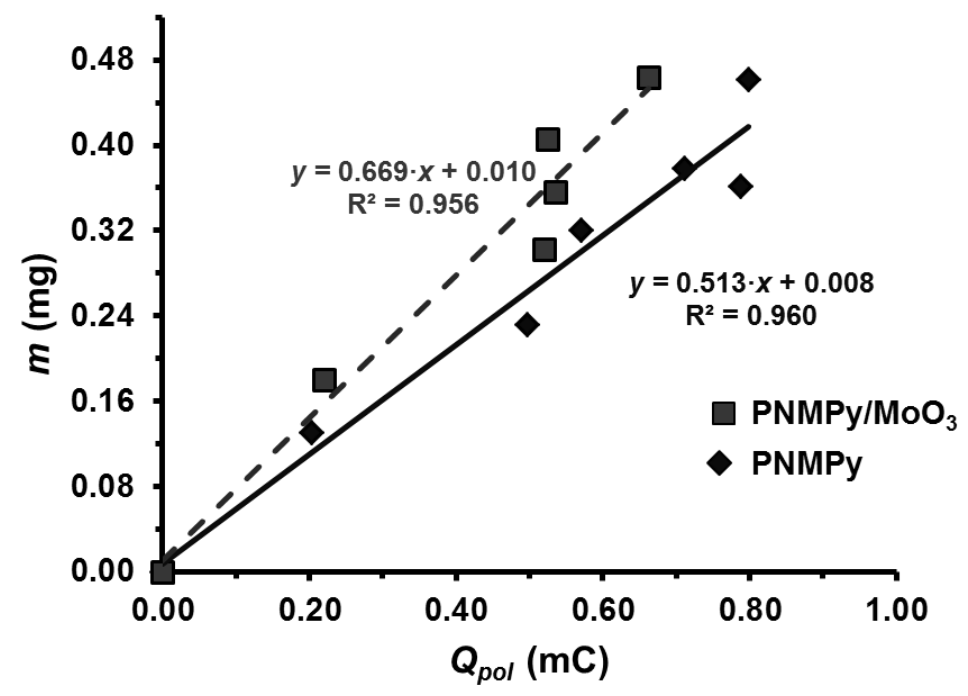

Figure 2 

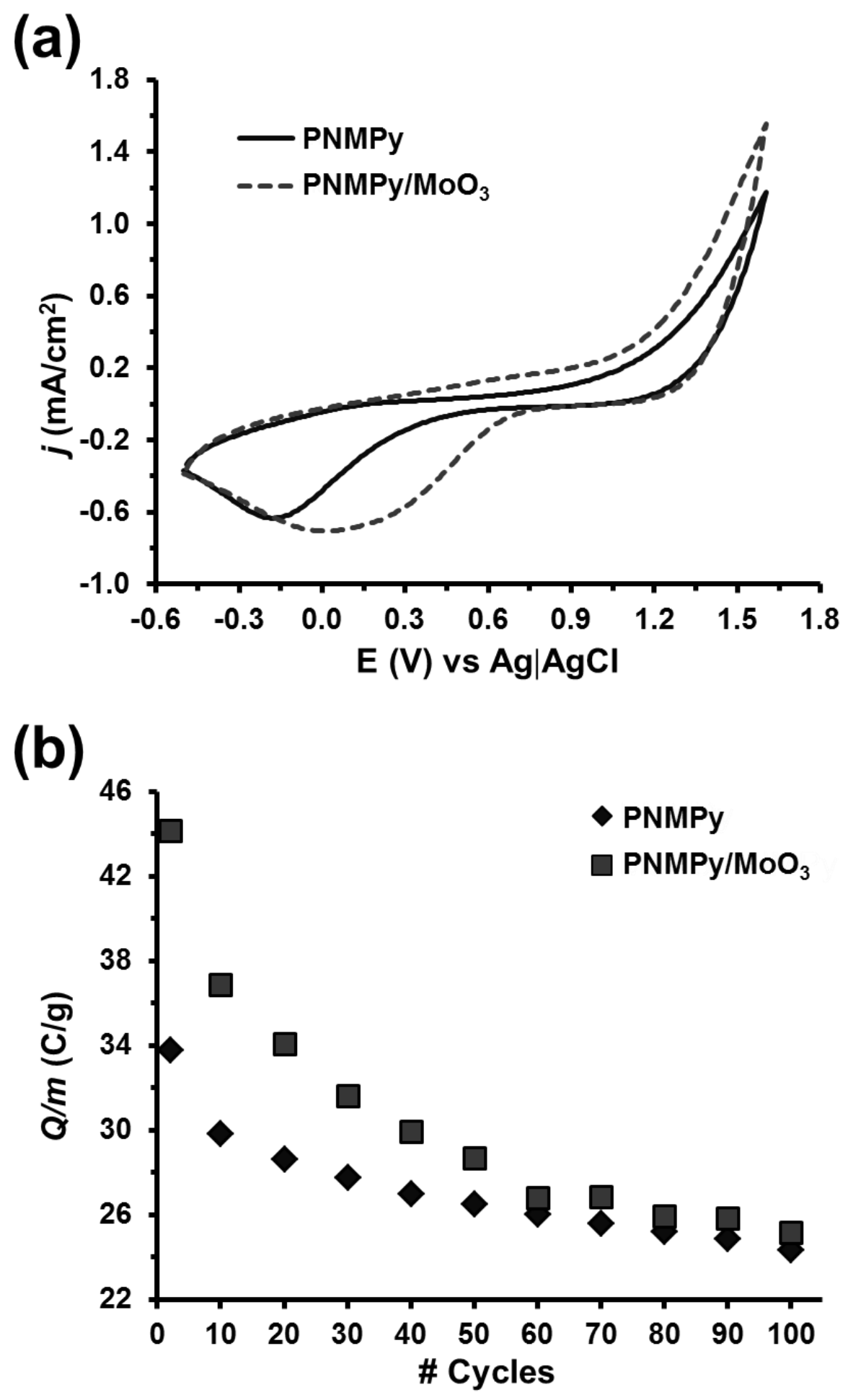

Figure 3 
(a)
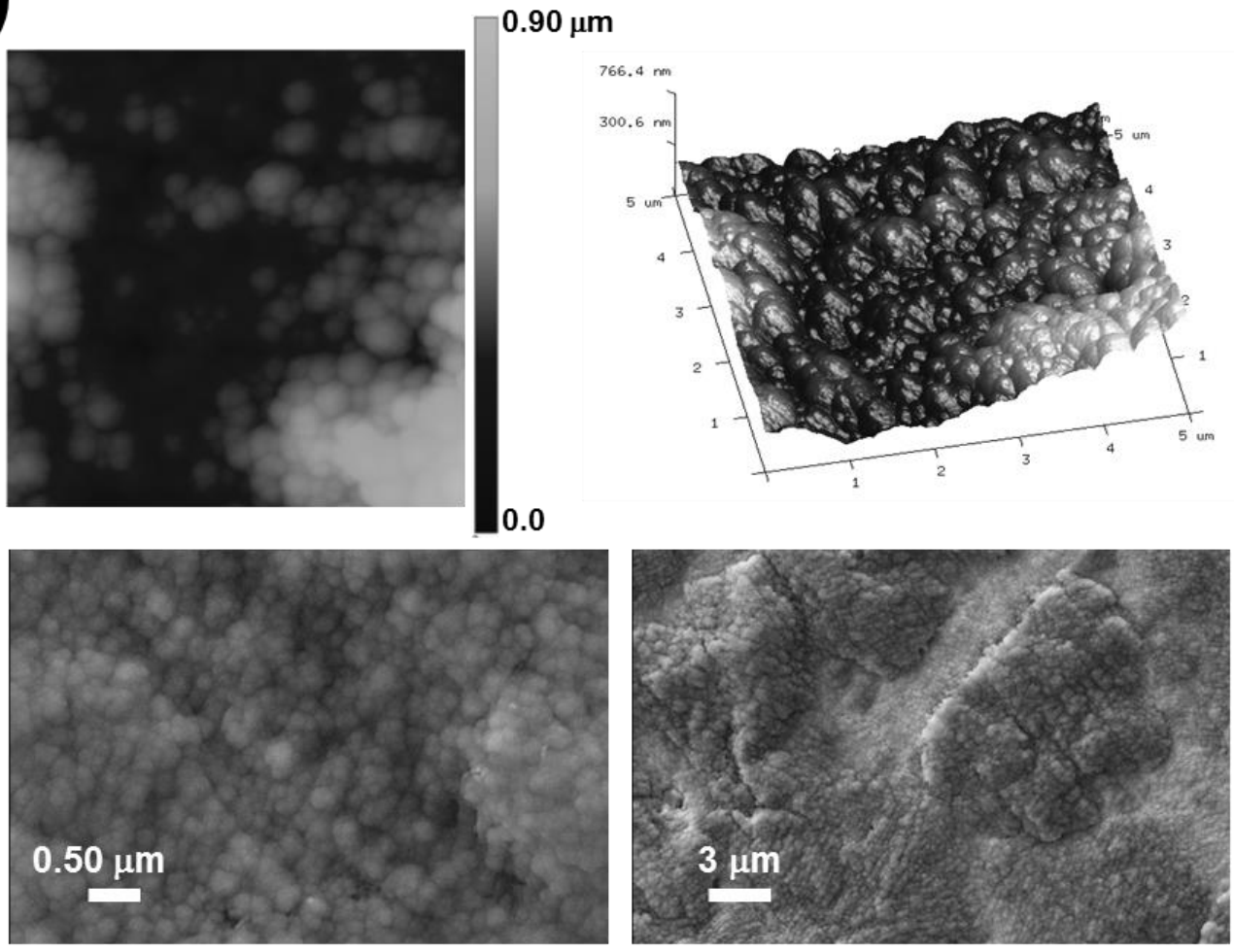

(b)

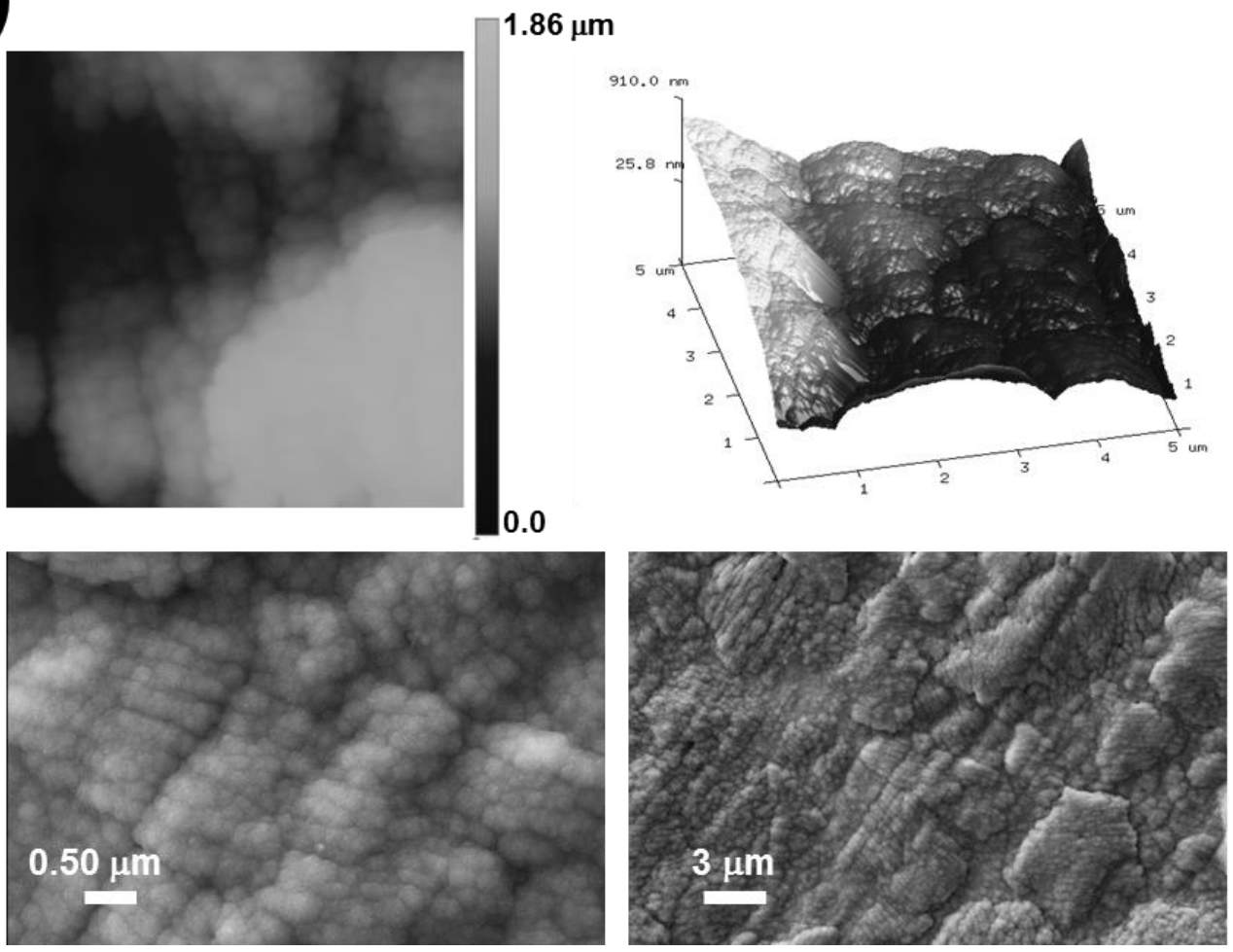

Figure 4 

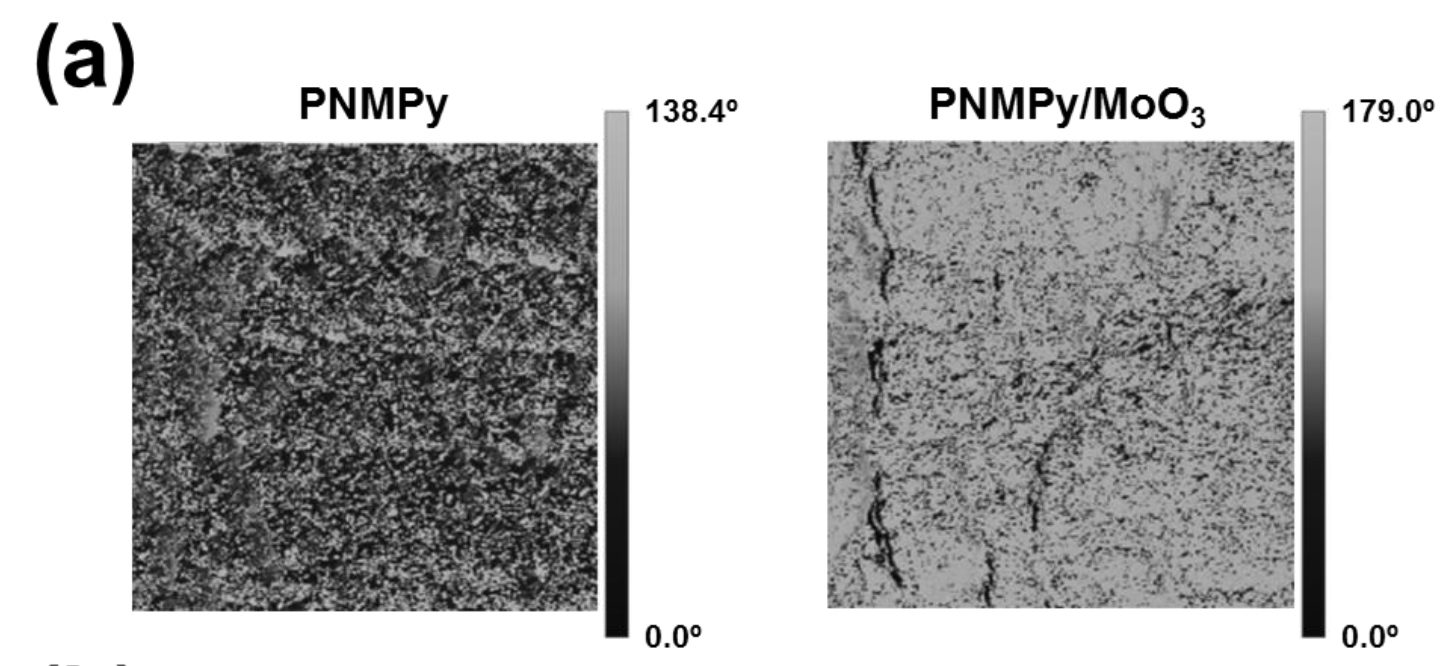

(b)

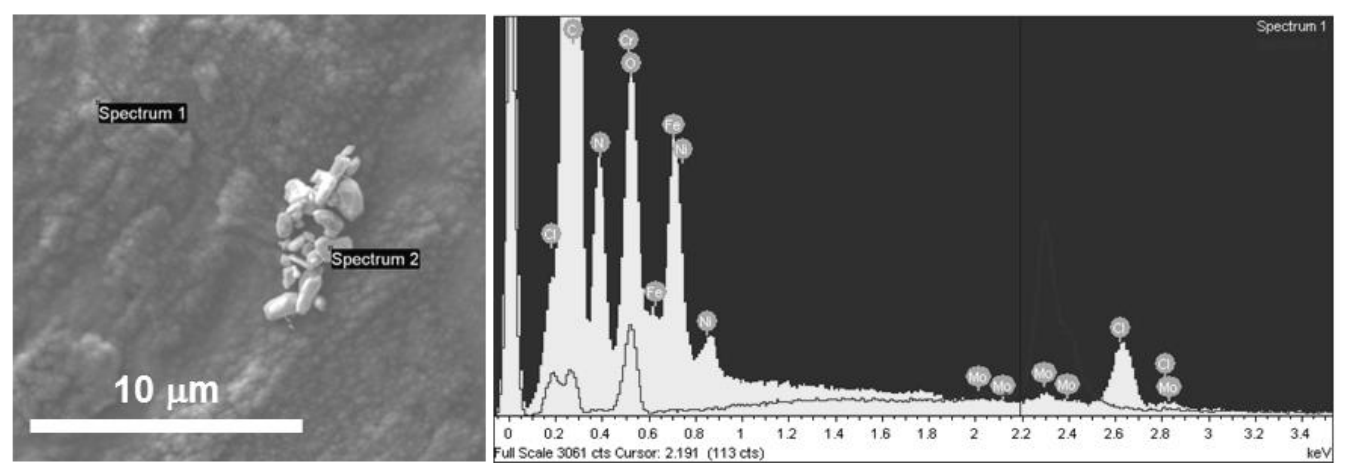

Figure 5 

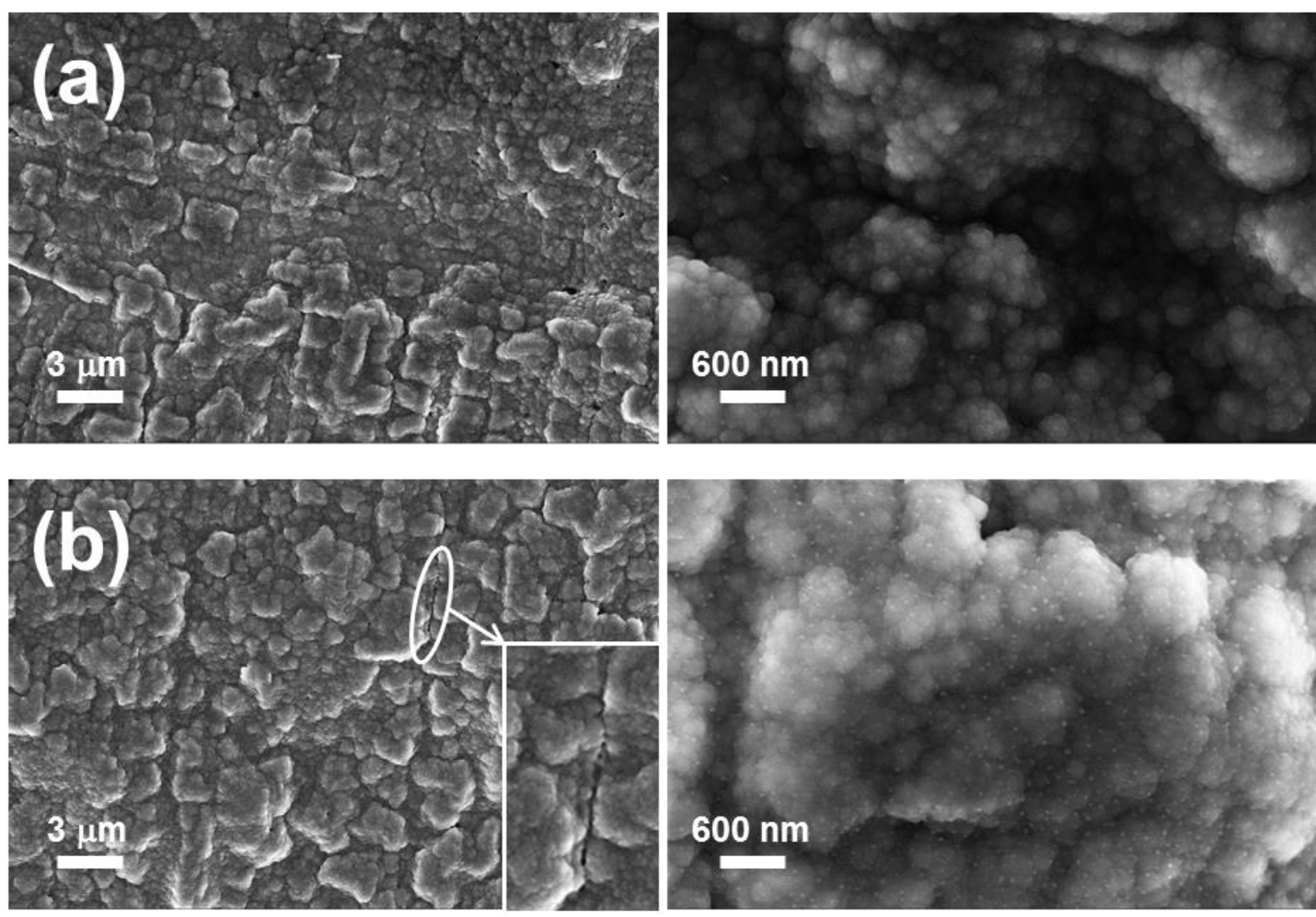

(c)

$600 \mathrm{~nm}$

Figure 6 